\title{
Magnetic manipulation
}

Our understanding of the neural correlates of behaviour has been advanced substantially in recent years by the development of optogenetic and chemogenetic tools. However, both of these techniques have limitations in vivo: optogenetics can be limited by poor light penetration into brain tissue, and chemogenetic tools can be limited by slow pharmokinetics. In this study, Wheeler and colleagues describe a non-invasive technique that uses a magnetic field to regulate the activity of neural circuits, both in vitro and in vivo.

It has been reported previously that transient receptor potential V1 (TRPV1) ion channels can be engineered to be activated by the combination of magnetothermal heating and exposure to radio waves. However, these approaches are complex and involve multiple components. Here, the authors developed a single component magnetogenetic system that they called 'Magneto2.0'. It was created by fusing TRPV4 with a magnetic field sensor, which in this case was the paramagnetic protein ferritin and was used to tug open the channel and induce depolarization.

Magneto2.0 was selectively expressed in excitatory entorhinal cortex neurons using Cre-loxmediated recombination. In brain slices, whole-cell current clamp of Magneto2.0-expressing neurons, concurrent with an applied $\sim 50 \mathrm{mT}$ static magnetic field, induced action potentials whose parameters (such as amplitude and action potential width) were similar to those produced by injection of $300 \mathrm{pA}$ of depolarizing current. The action potentials were blocked by prior incubation with the TRPV4 blocker GSK205, suggesting that the presence of Magneto2. 0 confers the ability to rapidly depolarize excitatory neurons by magnetic field stimulation.

The authors turned their attention to mice that had been transduced with a Magneto2.0-expressing construct selectively in the dopamine D1 receptor-expressing medium spiny neurons (MSNs) of the striatum, a large brain nucleus that is challenging to manipulate optogenetically because of the limited light penetration in the brain. Identifying MSNs based on their slow firing rate, the authors applied a magnetic field and found that there was an overall increase in the firing rate of these cells. The firing rate of striatal GABAergic cells that did not express Magneto2.0 remained unchanged during the magnetic stimulation period.

Systemic pharmacological activation of dopamine D1 receptor -expressing neurons in the striatum of mice evokes conditioned place preference. The authors expressed

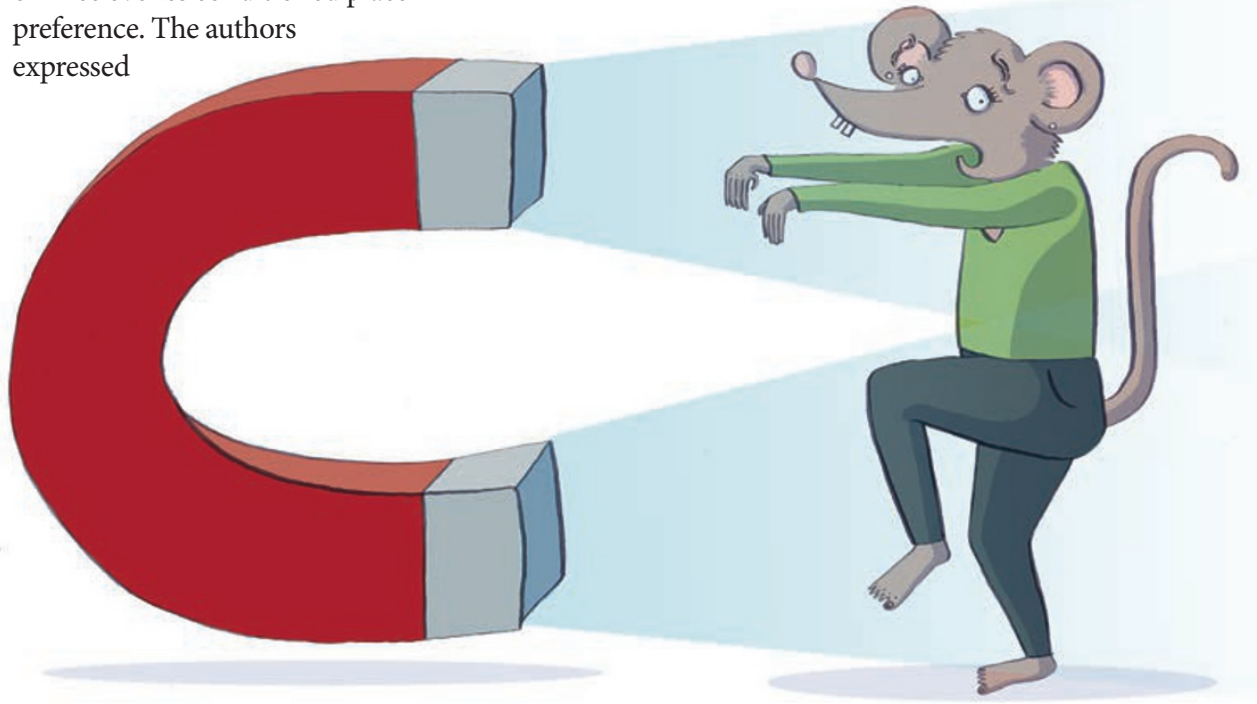

Magneto2.0 selectively in these neurons and placed the mice in a realtime place preference (RTPP) assay, in which mice could choose between two chambers, one of which had been magnetized using static magnetic fields generated by rare earth magnets. Mice expressing Magneto2.0 in D1 receptor-expressing striatal neurons showed a marked preference for the magnetized arm, which was lost when the magnets were removed.

Overall, these findings show that Magneto2.0 can be selectively expressed in specific neuronal populations, and that it can be used to remotely influence activity in these populations and in turn affect behaviour in brain regions that are difficult to access using other currently available techniques.

\section{Sian Lewis}

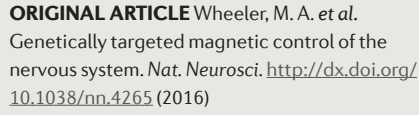

Magneto2.0

confers

the ability

to rapidly

depolarize

excitatory

neurons by

magnetic field

stimulation

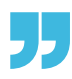

